\title{
Producción de ovinos de pelo bajo condiciones de pastoreo en el noreste de México
}

\author{
Production of hair SheeP Under grazing Conditions in nORTHEastern MeXico \\ Jairo Jeú Quintanilla-Medina ${ }^{1}$, Arnoldo González-Reyna ${ }^{1}$, \\ Javier Hernández-Meléndez ${ }^{1}$, Andrés Gilberto Limas-Martínez ${ }^{1}$, \\ Alejandro Carreón-Pérez ${ }^{1}$, Juan Carlos Martínez-González ${ }^{1,2}$
}

\section{Resumen}

El objetivo del estudio fue evaluar el efecto del genotipo, sexo, tipo de parto y año de nacimiento sobre el comportamiento productivo de corderos de razas de pelo (Blackbelly, Katahdin y Pelibuey) y cruces (Blackbelly x Dorper-Pelibuey) en un sistema de producción con pastoreo intensivo en la zona de Güémez, Tamaulipas, México. Se obtuvieron datos de 290 partos en cuatro épocas de parición y fueron analizados con un modelo lineal. El peso al nacimiento fue mayor en Katahdin $(3.75 \mathrm{~kg})$ y en corderos de parto simple (3.74 kg), mientras que el sexo del cordero no produjo diferencia significativa. La ganancia de peso predestete fue mayor en los corderos Blackbelly x Dorper-Pelibuey $(0.217 \mathrm{~kg})$, al igual que el peso al destete $(16.65 \mathrm{~kg})$.

Palabras clave: ovinos de pelo; peso al nacimiento; ganancia de peso; peso al destete

\section{Abstract}

The objective of the study was to evaluate the effect of genotype, sex, twins and year of birth on the productive behavior of purebred hair lambs (Blackbelly, Katahdin and Pelibuey) and crosses (Blackbelly x Dorper-Pelibuey) in a production system with intensive grazing in the area of Güémez, Tamaulipas, Mexico. Data from 290 lambings from four lambing seasons were analyzed with a linear model. Birth weight was greater in Katahdin

\footnotetext{
${ }^{1}$ Facultad de Ingeniería y Ciencias, Universidad Autónoma de Tamaulipas, Ciudad Victoria, Tamaulipas, México

${ }^{2}$ E-mail:jmartinez@docentes.uat.edu.mx
}

Recibido: 6 de octubre de 2017

Aceptado para publicación: 9 de febrero de 2018 
(3.75 kg) and in single lambing (3.74 kg) as compared to twin lambing, while the sex of the lamb did not show significant difference. The body weight gain at weaning was greater in the Blackbelly x Dorper-Pelibuey lambs $(0.217 \mathrm{~kg})$, as well as the weaning weight $(16.65 \mathrm{~kg})$.

Key words: hair sheep; body weight at birth; body weight gain; body weight at weaning

\section{INTRODUCCIÓN}

El inventario ovino en México ha crecido de 7.2 a 8.7 millones de cabezas en los últimos 10 años (SIAP, 2017). Esta reactivación de la ganadería ovina se ha visto además reflejada en una mayor integración de la producción primaria con los eslabones de transformación, procesamiento y comercialización de la cadena cárnica. Así mismo, en la diversificación de productos y subproductos ovinos, tales como cortes finos, carnes frías, embutidos, barbacoa y mixiotes enlatados. En Tamaulipas, el inventario se incrementó en más de 60000 cabezas entre 2006 y 2015 (SIAP, 2017), pero por otro lado, la producción de carne de ovino no satisface el consumo nacional teniendo que importarse el 43\% del requerimiento de países como Australia, Nueva Zelanda, Estados Unidos, Chile y Uruguay (SAGARPA, 2010).

La ovinocultura del país ha sido direccionada a la producción de carne en zonas tropicales, donde las razas de pelo sobresalen dada la capacidad de adaptación que presentan en dichos ambientes (García et al., 2004); de allí la importancia de caracterizar, evaluar y conservar los recursos genéticos disponibles. Magaña-Monforte et al. (2013) señalaron que un indicador de productividad que atañe a la oveja es la prolificidad, fertilidad y supervivencia, mientras que el cordero es responsable del peso al nacimiento, ganancia de peso y peso al destete. Sin embargo, estas características no han sido sustancialmente evaluadas bajo climas cálidos y húmedos en unidades de producción localizadas en el trópico mexicano.
Algunos estudios en México en sistemas con empadre continuo reportan un promedio de 231 días de intervalos entre partos en ovejas Blackbelly (Cadenas-Cruz et al., 2012), en tanto que González-Domínguez et al. (2016) señalan que el peso al nacimiento, ganancia diaria de peso predestete y peso al destete de corderos Blackbelly fueron de 2.9 $\pm 0.68 \mathrm{~kg}, 115 \pm 54 \mathrm{~g}$ y $14.8 \pm 5.6 \mathrm{~kg}$, respectivamente.

Por lo anterior, el objetivo fue evaluar algunos factores genéticos (Blackbelly, Katahdin, Pelibuey, Blackbelly x DorperPelibuey) y ambientales (sexo, tipo de parto, año de nacimiento y número de parto de la oveja) sobre el el comportamiento productivo de corderos en un sistema de producción con pastoreo intensivo en la zona de Tamaulipas, México.

\section{Materiales y MéTodos}

El trabajo se llevó a cabo en la Unidad de Producción Ovina de la Posta Zootécnica Herminio García González de la Facultad de Ingeniería y Ciencias de la Universidad Autónoma de Tamaulipas, localizada en el municipio de Güemez, Tamaulipas, México (INEGI, 2017).

Se analizaron 290 partos obtenidos en cuatro periodos de pariciones (julio de $2010 \mathrm{a}$ abril del 2012). Se hicieron lotes homogéneos según el genotipo de la oveja y de los sementales. Además, se consideró el sexo de la cría, número de crías por parto (simple, múltiple), año de parto y número de parto de 
la oveja (primípara, multípara). En cada periodo de empadre se conformaron cuatro grupos de ovejas, uno por cada genotipo racial materno (Blackbelly, Pelibuey, Katahdin y Dorper-Pelibuey $\left.\left[\mathrm{F}_{1}\right]\right)$, que fueron mantenidos en potreros separados con sementales puros Blackbelly, Katahdin y Pelibuey. Los cruces Dorper-Pelibuey fueron cubiertos con machos Blackbelly.

La alimentación del rebaño fue basada en pasturas y el uso de subproductos derivados de la industria citrícola (cáscara fresca de naranja). El periodo de pastoreo comprendió de marzo a diciembre de cada año y se utilizaron 6.0 ha de praderas irrigadas de Bermuda var. Tifton 68 (Cynodon nlemfuensis Vanderyst) y 85 (Cynodon spp), respectivamente, manejadas en forma rotacional, con 10 días de uso y 30 días de descanso. Después de cada pastoreo, las praderas fueron irrigadas por aspersión con una lámina de riego de $10 \mathrm{~cm}$. En el invierno (diciembre a marzo), las ovejas se alimentaron con cáscara fresca de cítricos (naranja y limón, principalmente) y suplementación proteica (soya y sorgo).

El programa sanitario establecido incluyó la vacunación y desparasitación dos veces al año (marzo y septiembre), considerándose el uso de Bacterina toxoide $(1.0 \mathrm{ml}$ animal $^{-1}$, Bayer, México) y antiparasitarios a base de ivermectina y closantel $(1.0 \mathrm{ml} 40$ $\mathrm{kg}$ de peso vivo ${ }^{-1}$, Avilab, México).

Durante el periodo de nacimientos, las ovejas fueron observadas por la mañana (08:00) y la tarde (18:00), para registrar los datos relacionados con el parto (número y sexo de los corderos). El peso de los corderos al nacimiento (PN) se determinó con una báscula colgante (Standar Scale, TECNOCOR 20-C, México) con capacidad de $20 \mathrm{~kg}$ y divisiones mínimas de $25 \mathrm{~g}$. La ganancia diaria de peso predestete (GDPP) se calculó con base en la relación GDPP = (peso al destete - peso al nacimiento) /días al destete. Cabe señalar que, dado el manejo del rebaño en la unidad de producción, el destete se realizó con base a factores relacionados con el cordero (peso) y la madre (condición corporal), para lo cual todas las crías fueron pesadas individualmente cada semana, además de observarse la condición corporal de las ovejas. El ajuste del peso se realizó a 60 días, utilizando la relación Peso ajustado a 60 días $=($ GDPP x 60$)+$ peso al nacer.

\section{Análisis Estadístico}

Los datos de cada variable se analizaron estadísticamente con un modelo lineal general (SAS, 2004), donde los factores en estudio fueron el grupo racial de los corderos (Pelibuey, Katahdin, Blackbelly, Blackbelly x Dorper-Pelibuey), el sexo de la cría (macho, hembra), el año de nacimiento $(2010,2011$, 2012), el tipo de parto (sencillo, múltiple) y el número de parto de la oveja (primípara, multípara).

El modelo estadístico fue $Y_{\mathrm{ijklmn}}=\mu+$ $\mathrm{GR}_{\mathrm{i}}+\mathrm{SX}_{\mathrm{j}}+\mathrm{AN}_{\mathrm{k}}+\mathrm{TP}_{1}+\mathrm{NP}_{\mathrm{m}}+\varepsilon_{\mathrm{ijklmn}}$, donde $\mathrm{Y}_{\mathrm{ijklmn}} \stackrel{\mathrm{j}}{=}$ variable dependiente de peso al nacer, ganancia diaria de peso predestete $y$ peso al destete ajustado a 60 días; $\mu=$ media general; $\mathrm{GR}_{\mathrm{i}}=$ grupo racial de los corderos; $\mathrm{SX}_{\mathrm{j}}=$ sexo del cordero; $\mathrm{AN}_{\mathrm{k}}=$ año de parto; $\mathrm{TP}_{1}=$ tipo de parto; $\mathrm{NP}_{\mathrm{m}}=$ número de parto de la oveja; y $\varepsilon_{\mathrm{ijklmn}}=$ error experimental.

Las interacciones de primer orden fueron eliminadas del modelo después de hacer varias corridas y no salir significativas. En aquellas variables donde resultaron significativos los efectos de tratamiento se realizó la prueba de comparación de medias de Tukey con una probabilidad de $\mathrm{p}=0.05$ (SAS, 2004).

\section{Resultados}

El peso al nacimiento (PN) fue afectado por el genotipo racial y el tipo de parto $(p<0.05)$, mientras que el sexo del cordero, año de parto y número de parto no produjeron diferencias significativas. En el Cuadro 1 se puede observar que los corderos Pelibuey, 
Cuadro 1. Efecto del genotipo racial sobre el peso al nacimiento en corderos de razas de pelo criadas bajo un sistema de pastoreo intensivo

\begin{tabular}{ccccc}
\hline & \multicolumn{3}{c}{ Número de partos por año } & \multirow{2}{*}{$\begin{array}{c}\text { Peso al nacimiento } \\
(\mathrm{kg})\end{array}$} \\
\cline { 2 - 4 } & 2010 & 2011 & 2012 & \\
\hline Genotipo racial & 17 & 37 & 14 & $3.24 \pm 0.92^{\mathrm{b}}$ \\
Blackbelly (BB) & 14 & 27 & 54 & $3.57 \pm 0.76^{\mathrm{ab}}$ \\
BB x Dorper-PB & 53 & 20 & 7 & $3.75 \pm 1.05^{\mathrm{a}}$ \\
Katahdin & 15 & 17 & 15 & $3.37 \pm 1.06^{\mathrm{b}}$ \\
Pelibuey (PB) & & & & \\
Tipo de parto & 60 & 40 & 64 & $3.74 \pm 1.01^{\mathrm{a}}$ \\
Sencillo & 38 & 61 & 27 & $3.16 \pm 0.83^{\mathrm{b}}$ \\
Múltiple & & & & \\
\hline
\end{tabular}

a,b Valores con distinta letra dentro de columnas son estadísticamente diferentes $(p<0.05)$

Cuadro 2. Efecto del genotipo racial sobre la ganancia diaria de peso predestete (GDPP) y peso ajustado al destete (PDA) de corderos de razas de pelo, en un sistema de pastoreo intensivo

\begin{tabular}{lcc}
\hline Genotipo racial & GDPP $\left(\mathrm{kg} \mathrm{día} \mathrm{a}^{-1}\right)$ & PDA $(\mathrm{kg})$ \\
\hline Blackbelly $(\mathrm{BB})$ & $0.155 \pm 0.05^{\mathrm{b}}$ & $12.62 \pm 3.27^{\mathrm{b}}$ \\
BB x Dorper-PB & $0.217 \pm 0.06^{\mathrm{a}}$ & $16.65 \pm 4.17^{\mathrm{a}}$ \\
Katahdin & $0.170 \pm 0.07^{\mathrm{b}}$ & $14.04 \pm 4.61^{\mathrm{b}}$ \\
Pelibuey (PB) & $0.173 \pm 0.07^{\mathrm{b}}$ & $13.76 \pm 4.67^{\mathrm{b}}$ \\
\hline
\end{tabular}

a,b Valores con distinta letra dentro de columnas son estadísticamente diferentes $(p<0.05)$

Blackbelly puro y Blackbelly x DorperPelibuey presentaron un PN similar entre sí, pero inferior al observado en las crías Katahdin. El PN de los corderos provenientes de parto simple fue mayor $(\mathrm{p}<0.05)$ que en las crías de parto múltiples (Cuadro 1).

El análisis de la ganancia diaria de peso predestete (GDPP) mostró que solamente el genotipo racial y el año de nacimiento produjeron comportamientos distintos $(p<0.05)$. En el Cuadro 2 se puede observar que los corderos Blackbelly x Dorper-Pelibuey presentaron mayor ganancia de peso predestete $(\mathrm{p}<0.05)$ que los Katahdin, Pelibuey y
Blackbelly puros. Además, las ovejas en dicho grupo genético tuvieron una mayor proporción de partos simples (1.21 corderos por parto). La GDPP entre los grupos Katahdin, Pelibuey y Blackbelly puros fue similar. Por otro lado, el GDPP de los corderos nacidos en 2010, 2011 y 2012 fue de $236 \pm 4,184 \pm 7$ y $121 \pm 4 \mathrm{~g}$, respectivamente $(\mathrm{p}<0.05)$.

Los corderos nacidos en partos simples ganaron mayor peso en el periodo predestete, ventaja que fue más notable en los Katahdin, con incrementos de $43.7 \%$ más peso que los de parto múltiple, mientras que las diferencias fueron de $9.8,4.7$ y $1.7 \%$ en Blackbelly 
x Dorper-Pelibuey y Blackbelly y Pelibuey puros, respectivamente. El sexo del cordero no produjo diferencias significativas para la GDPP ni para el peso al destete ajustado.

El peso al destete ajustado solamente fue afectado $(\mathrm{p}<0.05)$ por el genotipo racial (Cuadro 2) y el año de nacimiento. Los corderos Blackbelly x Dorper-Pelibuey fueron los que alcanzaron el mayor PDA (16.65 \pm $4.17 \mathrm{~kg})$.

\section{Discusión}

Los corderos más pesados al nacimiento fueron los Katahdin, probablemente debido a la mayor talla y capacidad cárnica de la raza (Burke y Apple, 2007; Lucero-Magaña et al., 2011; Mellado et al., 2016), pero sin que al peso al nacer fuera diferente de los Blackbelly x Dorper-Pelibuey $\left(\mathrm{F}_{1}\right)$, donde contribuye otra raza especializada (Dorper) en el genotipo. El comportamiento diferencial entre los corderos fue definido por las características propias de las razas que los conformaron; además de factores como peso de la madre, producción de leche y habilidad materna (Burke y Apple, 2007; Macias-Cruz et al., 2012). Los valores observados se encuentran dentro del rango reportado en la literatura en corderos Pelibuey, Dorper, Katahdin y cruces (Burke, 2005; HinojosaCuellar et al., 2009).

El peso al nacimiento de los corderos Pelibuey fueron superiores a los reportados por Gutiérrez et al. (2005), quienes señalan que el bajo peso al nacer, crecimiento y calidad de la canal de estos corderos son inferiores a los observados en razas de lana o cárnicas. En este sentido Macias-Cruz et al. (2010) observaron que los cruzamientos entre hembras Pelibuey y sementales Dorper o Katahdin producen corderos para el abasto que presentan tasas de crecimiento superiores a los Pelibuey puros, así como buena adaptación en climas áridos. En el caso de los corderos Blackbelly, el peso al nacimiento fue superior al $2.7 \mathrm{~kg}$ reportado por GonzálezGarduño et al. (2002) y González-Domínguez et al. (2016) cuando las madres fueron mantenidas en un ambiente de trópico húmedo.

El peso al nacer de corderos provenientes de parto simple fue mayor, como se reporta en la literatura (González-Domínguez et al., 2016), debido entre otros factores a la menor competencia en el útero por nutrientes y espacio (González-Garduño et al., 2002). La similutud del peso al nacer entre sexos fue coincidente con el estudio de Macedo y Arredondo (2008); no obstante, Ferro et al. (2011) observaron en ovinos Pelibuey que los machos presentan mayor peso al nacimiento que las hembras.

La ganancia diaria de peso predestete (GDPP) fue afectada por el genotipo racial y el año de nacimiento. Este comportamiento es atribuible a que los corderos Blackbelly $\mathrm{x}$ Dorper-Pelibuey poseían rasgos genéticos de una raza cárnica, tal y como lo señala MaciasCruz et al. (2012) en su estudio con corderos Dorper x Pelibuey, Katahdin x Pelibuey y Pelibuey puros. Por otro lado, HinojosaCuéllar et al. (2009, 2013) reportan una GDPP entre 139 y $160 \mathrm{~g}$ en corderos de varias razas de pelo, mientras que GonzálezGarduño et al. (2002) observaron ganancias de $122 \mathrm{~g} \mathrm{día}^{-1}$ en corderos híbridos de razas de pelo $\mathrm{x}$ razas de lana.

Los corderos nacidos en partos simples tuvieron mayor GDPP, ventaja que fue más notable en la raza Katahdin, donde los corderos nacidos en parto simple ganaron un $43.7 \%$ más peso que los de parto múltiple, aspecto que fue observado por Hinojosa-Cuéllar et al. (2013) en corderos de las razas $\mathrm{F}_{1}$ Pelibuey $x$ Blackbelly y cruces con Dorper y Katahdin. Asimismo, la variación en la GDPP entre años pudo deberse a diferencias en el ambiente y nivel nutricional (García et al., 2004). Es así que la mayor GDPP en este estudio fue superior a la reportada por Hinojosa-Cuéllar $e t$ al. (2009), lo que se debió a una mejor disponibilidad y calidad del alimento. 
El sexo del cordero no produjo diferencia significativa para la GDPP, tal y como lo reportan González-Garduño et al. (2010). Asimismo, el peso al destete ajustado (PDA) solamente fue afectado por el genotipo racial y el año de nacimiento. Dicho comportamiento es atribuido al esquema de cruzamientos realizado en este estudio, donde los corderos poseen rasgos genéticos especializados en producción de carne (Macías-Cruz et al., 2012). Sin embargo, Hinojosa-Cuellar et al. (2013) observaron que los corderos $F_{1}$ fueron más productivos que los que tenían genes de las razas Dorper o Katahdin.

El peso al destete ajustado $(14.27 \mathrm{~kg})$ registrado en el presente estudio es comparable a los $13.7 \mathrm{~kg}$ de peso en corderos Blackbelly destetados a 90 d (GonzálezGarduño et al., 2002). Una mayor variación en el peso al destete (11-17 kg) fue informada por Hinojosa-Cuéllar et al. (2009, 2013). Las diferencias en la alimentación preparto y posparto de las ovejas, así como la cantidad y calidad del complemento alimenticio contribuyen a explicar la variación en peso al destete ajustado. Por otro lado, este peso no fue afectado por el número de parto de la oveja, aunque González-Domínguez et al. (2016) mencionan que el peso al destete de corderos Blackbelly fue afectado por el número de partos de la madre.

\section{Conclusiones}

- El genotipo racial y el tipo de parto son factores determinantes en la expresión del peso al nacimiento de corderos en sistemas intensivos en pastoreo.

- La ganancia diaria de peso predestete y el peso al destete de los corderos fueron afectados por el genotipo, obteniéndose un mejor comportamiento en corderos con genotipos especializados en producción de carne.

\section{Agradecimientos}

Los autores desean expresar su agradecimiento al CONACYT por el financiamiento para cursar estudios de maestría del primer autor (266121).

\section{Literatura Citada}

1. Burke J, Apple J. 2007. Growth performance and carcass traits of foragefed hair sheep wethers. Small Ruminant Res 67:264-270. doi: 10.1016/j.smallrumres.2005.10.014

2. Burke J. 2005. Lamb production of Dorper, Katahdin, and St. Croix bred in summer, winter, or spring in the Southeastern United States. Sheep Goat Res J 20: 51-59.

3. Cadenas-Cruz PJ, Oliva-Hernández J, Hinojosa-Cuellar JA. 2012. Productivity of Blackbelly ewes and their hybrid litter under grazing. J Anim Vet Adv 11:97-102.

4. Ferro M, Pulgarón P, Lleimys C. 2011. Influencia de factores no genéticos en el comportamiento productivo de corderos Pelibuey. REDVET 12(2). [Internet]. Disponible en: http://www.veterinaria.org/revistas/redvet/n020211/ 021102.pdf

5. García J, Miñon D, Álvarez M, Giorgetti H, Rodríguez G, Perlo A. 2004. Cruzamientos industriales para producción de carne ovina. IDIA XXI: 159-162.

6. González-Domínguez G, HinojosaCuéllar JA, Oliva-Hernández J, Torres-Hernández G, Segura-Correa $J C$, González-Garduño R, GarcíaOsorio IC. 2016. Análisis del crecimiento predestete de corderos Barbados Barriga Negra en clima cálido húmedo. Nova Scentia 8: 181-197.

7. González-Garduño $R$, TorresHernández G, Arece-García J. 2010. Comportamiento productivo y 
reproductivo de ovinos Pelibuey en un sistema de pariciones aceleradas con tres épocas de empadre al año. Zootecnia Trop 28: 179-187.

8. González-Garduño $R$, TorresHernández G, Castillo-Álvarez M. 2002. Crecimiento de corderos Blackbelly entre el nacimiento y el peso final en el trópico húmedo de México. Vet Méx 33: 443-453.

9. Gutiérrez, J, Rubio S, Méndez, D. 2005. Effects of crossbreeding Mexican Pelibuey sheep with Rambouillet and Suffolk on carcass traits. Meat Sci 70: 1-5. doi: 10.1016/j.meatsci.2004.10.017

10. Hinojosa-Cuéllar JA, OlivaHernández J, Torres-Hernández G, Segura-Correa JC. 2013. Comportamiento productivo de corderos $\mathrm{F} 1$ Pelibuey x Blackbelly y cruces con Dorper y Katahdin en un sistema de producción del trópico húmedo de Tabasco, México. Arch Med Vet 45: 135-143. doi: 10.4067/S0301-732X2013000200004

11. Hinojosa-Cuéllar JA, RegaladoArrazola F, Oliva-Hernández J. 2009. Crecimiento prenatal y predestete en corderos Pelibuey, Dorper, Katahdin y sus cruces en el sureste de México. Rev Cient LUZ 19: 522-532.

12. [INEGI] Instituo Nacional de Geografía y Estadística. 2017. Güémez, Tamaulipas. [Internet]. Disponible en: http://www.beta.inegi.org.mx/app/mapa/ espacioydatos/default.aspx?ag=28013

13. Lucero-Magaña H, Briones-Encinia F, Lucero-Magaña FA, HernándezMeléndez J, Castillo-Rodríguez SP, Martínez-González JC. 2011. Estrategias para incrementar la producción de carne de ovinos de pelo en la Huasteca Potosina, México. Zootecnia Trop 29: 255-260.

14. Macedo R, Arredondo V. 2008. Efecto del sexo, tipo de nacimiento y lactancia sobre el crecimiento de ovinos Pelibuey en manejo intensivo. Arch Zootec 57: 219-228.
15. Macías-Cruz U, Álvarez-Valenzuela FD, Rodríguez-García J, Correa-Calderón A, Torrentera-Olivera NG, Molina-Ramírez L, Avendaño-Reyes L. 2010. Crecimiento y características de canal en corderos Pelibuey puros y cruzados F1 con razas Dorper y Katahdin en confinamiento. Arch Med Vet 42: 147-154.

16. Macías-Cruz U, Álvarez-Valenzuela FD, Olguín-Arredondo HA, MolinaRamírez L, Avendaño-Reyes L. 2012. Ovejas Pelibuey sincronizadas con progestágenos y apareadas con machos de razas Dorper y Katahdin bajo condiciones estabuladas: Producción de la oveja y crecimiento de los corderos durante el periodo predestete. Arch Med Vet 44: 29-37. doi: 10.4067/S0301732X2012000100005

17. Magaña-Monforte JG, Huchin-Cab M, Ake-López RJ, Segura-Correa JC. 2013. A field study of reproductive performance and productivity of Pelibuey ewes in Southeastern Mexico. Trop Anim Health Prod 45: 1771-1776. doi: 10.1007/ s11250-013-0431-2

18. Mellado, M, Macías U, Avendaño L, Mellado J, García JE. 2016. Growth and pre-weaning mortality of Katahdin lamb crosses. Rev Colomb Cienc Pec 29: 288-295.

19. [SAGARPA] Secretaria de Agricultura Ganadería, Desarrollo Rural, Pesca y Alimentación. 2010. Estimación del consumo nacional aparente 19902005. [Internet]. Disponible en: http:// www.sagarpa.gob.mx/ganaderia/ Estadisticas/Lists/Estadsticas/ Attachments/6/Estimaci\%C3\%B3n\%20de1\%20-Consumo\%20Nacional\%20Aparente\%201990-2005\%20Miel$\% 20 \mathrm{de} \% 20$ abeja.pdf

20. [SAS] Statistical Analysis System. 2004. SAS/STAT 9.1 User's guide: basics. Institute Statistical Analysis System. Cary, NC, USA. [Internet]. Disponible en: https://support.sas.com/ 
documen-tation/onlinedoc/91pdf/ sasdoc 91/stat_ug 7313.pdf

21. [SIAP] Servicio de Información Agroalimentaria y Pesquera. 2017. Secretaría de Agricultura Ganadería, Desarrollo Rural, Pesca y Alimentación.
Resumen Nacional. Población Ganadera Ovina 2005-2015. [Internet]. Disponible en: https://www.gob.mx/cms/ uploads/attachment/file/166001/ ovino.pdf 